\title{
OBTENÇÃO DE EXTRATOS DE TANSAGEM (Plantago major) POR EXRAÇÃ̃O SUPERCRÍTICA E EXTRAÇÃO COM SOLVENTES ORGÂNICOS
}

\author{
S. MAZZUTTI ${ }^{1}$ e S. R. S. FERREIRA ${ }^{1}$ \\ ${ }^{1}$ Universidade Federal de Santa Catarina, Departamento de Engenharia Química e Engenharia de \\ Alimentos \\ E-mail: sandra@enq.ufsc.br
}

\begin{abstract}
RESUMO - A tansagem (Plantago major) tem sido usada desde os tempos antigos como um recurso de medicina alternativa para o tratamento de diversas enfermidades. $\mathrm{O}$ presente estudo objetivou a obtenção de extratos de tansagem ( $P$. major) por diferentes métodos: Soxhlet (SOX) e ultrassom (US) empregando diferentes solventes ou misturas de solventes e extração supercrítica (ESC) com dióxido de carbono e adicionado de etanol como cossolvente em diferentes concentrações. As técnicas de extração foram comparadas em termos de rendimento de processo. Nas extrações à baixa pressão, o maior rendimento $(20,5 \pm 0,8)$ foi obtido por ultrassom empregando a mistura de etanol/água (50/50) como solvente. O maior rendimento de extração por ESC foi obtido quando utilizado $10 \%$ de etanol como modificador, atingindo rendimento de $2,27 \pm 0,07 \%$.
\end{abstract}

\section{INTRODUÇÃO}

A busca por compostos bioativos naturais com potencial tanto para o tratamento e prevenção de doenças humanas, bem como para satisfazer outras necessidades é atualmente um tema chave em muitos laboratórios e indústrias (Gil-Chávez et al., 2013). Quando se procura obter substâncias ativas de plantas, um dos principais aspectos que deve ser observado consiste nas informações da medicina popular. Dados da literatura revelam que é muito mais provável encontrar atividade biológica em plantas orientadas pelo seu uso na medicina popular do que em plantas escolhidas ao acaso (Cechinel Filho e Yunes, 1998).

A Plantago major tem sida usada desde os tempos antigos como um recurso de medicina alternativa. A planta vegeta espontaneamente em áreas de pastagens, terrenos cultivados e locais úmidos e sombreados, sendo considerada invasora de culturas (Samuelsen, 2000). No Brasil, a $P$. major é conhecida como tansagem, transagem e tanchagem. O chá de tansagem (P. major) é usado como depurativo, diurético, antibacteriano, regulador intestinal e para tosses, bronquites, dor de estômago, diarreia e disenteria. Em gargarejos a tansagem é indicada para combater dor e inflamação da garganta. As folhas frescas maceradas possuem propriedades cicatrizantes, sendo úteis contra picadas de insetos, irritações da pele, úlceras, queimaduras e pequenos cortes (Blanco et al., 2008, CháECia, 2013). 


\section{9 a 22 de outubro de 2014 \\ Florianópolis/SC}

Os métodos comumente utilizados para a extração de compostos ativos de plantas são a extração líquido-líquido ou sólido-líquido. No entanto, as dificuldades inerentes na obtenção desses compostos conduziram ao desenvolvimento de tecnologias avançadas, como a extração com líquido pressurizado, extração subcrítica e supercrítica, extração assistida por ultrassom e micro-ondas (GilChávez et al., 2013).

A tecnologia supercrítica, que utiliza solventes a alta pressão, é considerada um processo limpo, sendo que os extratos obtidos através desta técnica apresentam alta pureza. Este processo apresenta vantagens quando comparado com outras técnicas de separação, a saber: compostos termicamente instáveis podem ser separados a baixas temperaturas; o solvente pode ser removido facilmente do soluto por redução de pressão e/ou ajuste de temperatura; a necessidade de energia térmica é inferior à do processo de destilação; alta seletividade para os solutos; rápida extração devido à baixa viscosidade do solvente, alta difusividade e bom poder de solvatação do solvente (Brunner, 1994).

No que diz respeito a técnicas que envolvem o uso de fluidos supercríticos, o dióxido de carbono $\left(\mathrm{CO}_{2}\right)$ é sem dúvida o solvente mais empregado. $\mathrm{O} \mathrm{CO}_{2}$ apresenta diversas vantagens que decorrem do fato de ser um solvente barato, não inflamável, sem risco de explosão e quimicamente inerte, produzindo um extrato vegetal com alta pureza. O maior obstáculo ao uso da extração supercrítica na indústria ainda é o alto custo fixo de investimento necessário para a instalação de uma planta de extração (Pereira e Meireles, 2010).

Esse trabalho teve como objetivo obter extratos de tansagem (P. major) por extração supercrítica e extração com diferentes solventes orgânicos, avaliando as técnicas em termos de rendimento de processo.

\section{MATERIAL E MÉTODOS}

\subsection{Preparo da amostra}

A amostra de tansagem (P. major) utilizada para as extrações foi adquirida junto a empresa Chá \& Cia Ervas Medicinais, sendo composta pelas partes aéreas da planta. A tansagem ( $P$. major) foi produzida no Brasil, colhida em agosto de 2012, seca em estufa e comercializada com teor de umidade de $12,05 \%$ e teor de cinzas de $12,52 \%$. A amostra foi moída em moinho tipo Willey, empacotada em saco plástico identificado e armazenada em congelador doméstico a $-18{ }^{\circ} \mathrm{C}$.

\subsection{Extrações com solventes orgânicos}

Soxhlet: A extração Soxhlet foi realizada de acordo com o método 920.39C da AOAC (2005), sendo utilizados $5 \mathrm{~g}$ de amostra, $150 \mathrm{~mL}$ de solvente e tempo de extração de $6 \mathrm{~h}$. Nas extrações com P. major utilizaram-se os solventes orgânicos hexano, diclorometano, acetato de etila, etanol e metanol. A eliminação dos solventes foi realizada em rotaevaporador, sob vácuo de $400 \mathrm{mmHg} \mathrm{e}$ rotação de $50 \mathrm{rpm}$. Os extratos foram acondicionados em frascos âmbar, armazenados em congelador doméstico a $-18^{\circ} \mathrm{C}$ e os resultados expressos como média \pm desvio padrão. 


\section{9 a 22 de outubro de 2014 \\ Florianópolis/SC}

Ultrassom: A extração assistida por ultrassom da $P$. major foi realizada em acordo com Andrade et al. (2012), sendo utilizados $7 \mathrm{~g}$ de amostra, $210 \mathrm{~mL}$ de solvente e tempo de extração de 45 min. Os solventes orgânicos utilizados foram: hexano, diclorometano, acetato de etila, etanol e metanol e duas misturas etanol/água nas proporções 50/50 e 70:30 (v/v). A eliminação dos solventes foi realizada em rotaevaporador, sob vácuo de $400 \mathrm{mmHg}$ e rotação de $50 \mathrm{rpm}$. Os extratos foram acondicionados em frascos âmbar, armazenados em congelador doméstico a $-18^{\circ} \mathrm{C}$ e os resultados expressos como média \pm desvio padrão.

\subsection{Extração com solvente supercrítico (ESC)}

As extrações foram realizadas utilizando $15 \mathrm{~g}$ de amostra, vazão de dióxido de carbono de $8 \pm 2$ $\mathrm{g} / \mathrm{min}$ e tempo de extração de $3,5 \mathrm{~h}$. A influência da pressão e da temperatura no rendimento global $\left(\mathrm{X}_{0}\right)$ da ESC da tansagem ( $P$. major) foi avaliada por meio de um planejamento experimental com 3 níveis e 2 variáveis, totalizando 9 experimentos. Nos experimentos, realizados em duplicata, a temperatura variou entre 40 e $60{ }^{\circ} \mathrm{C}$ e a pressão entre 10 e $30 \mathrm{MPa}$. O limite inferior de temperatura $\left(40{ }^{\circ} \mathrm{C}\right)$ está um pouco acima da temperatura crítica do $\mathrm{CO}_{2}\left(31,1^{\circ} \mathrm{C}\right)$ e é normalmente utilizada na extração de materiais vegetais nos processos de ESC. O limite superior de temperatura $\left(60{ }^{\circ} \mathrm{C}\right)$ permite a extração de compostos de materiais vegetais e não causa danos térmicos no material.

O emprego de cossolvente foi avaliado na condição de $50{ }^{\circ} \mathrm{C}$ e $30 \mathrm{MPa}$, utilizando $15 \mathrm{~g}$ de amostra, vazão de dióxido de carbono de $8 \pm 2 \mathrm{~g} / \mathrm{min}$ e tempo de extração de $3,5 \mathrm{~h}$. O cossolvente escolhido foi o etanol, sendo adicionado nas frações de $0 ; 2,5 ; 5$ e $10 \%$. A eliminação dos solventes foi realizada em rotaevaporador, sob vácuo de $400 \mathrm{mmHg}$ e rotação de $50 \mathrm{rpm}$. Todos os extratos foram acondicionados em frascos âmbar, armazenados em congelador doméstico a $-18^{\circ} \mathrm{C}$ e os resultados expressos como média \pm desvio padrão.

\subsection{Análise estatística}

Os resultados de rendimento global foram avaliados estatisticamente por análise unidirecional de variância (ANOVA). A análise estatística foi realizada para detectar diferenças significativas entre os valores de rendimento em função da temperatura e pressão na ESC As diferenças significativas ( $p$ $<0,05)$ foram analisadas pelo teste de Tukey.

\section{RESULTADOS E DISCUSSÕES}

Os rendimentos dos extratos de tansagem (P. major) obtidos com diferentes solventes orgânicos em Soxhlet e ultrassom estão apresentados na Tabela 1. Os resultados de rendimento apresentaram diferenças significativas entre si, variando entre 1,46 \% para a extração em ultrassom com acetato de etila e 20,5 \% para a extração em ultrassom com a mistura etanol/água (50/50). Gálvez et al. (2005) estudaram a atividade antioxidante de extratos de espécies de Plantago obtidos em Soxhlet. Os rendimentos verificados pelos autores das extrações em Soxhlet empregando metanol por $48 \mathrm{~h}$ variaram entre $18,04 \%$ para $P$. afra e $29,13 \%$ para $P$. lanceolata.

Comparando-se as técnicas de extração para um mesmo solvente pode-se verificar que maiores 
rendimentos foram obtidos quando utilizada a técnica Soxhlet. Nessa técnica, a amostra está sempre em contato com o solvente, havendo sua constante renovação, o que não ocorre no processo de extração em ultrassom. Além disso, o tempo e a temperatura de extração da técnica Soxhlet foram superiores. A ação conjunta desses fatores podem ter proporcionado uma extração mais exaustiva das amostras submetidas a técnica de Soxhlet em comparação ao ultrassom, resultando em maiores rendimentos.

Tabela 1 - Rendimento global $\left(\mathrm{X}_{0}\right)$ obtidos para as extrações com solventes orgânicos de tansagem (P. major) com diferentes solventes.

\begin{tabular}{lcc}
\hline Técnica & Solvente & Rendimento $(\%)^{(1)}$ \\
\hline \multirow{3}{*}{ Soxhlet } & Hexano & $3,9 \pm 0,2^{\mathrm{de}}$ \\
& Diclorometano & $2,13 \pm 0,09^{\mathrm{dg}}$ \\
& Acetato de etila & $3,31 \pm 0,03^{\mathrm{df}}$ \\
& Etanol & $11,6 \pm 0,1^{\mathrm{c}}$ \\
& Metanol & $18,3 \pm 1,2^{\mathrm{ab}}$ \\
\hline \multirow{3}{*}{ Ultrassom } & Hexano & $1,5 \pm 0,2^{\mathrm{efg}}$ \\
& Diclorometano & $1,58 \pm 0,05^{\mathrm{efg}}$ \\
& Acetato de etila & $1,46 \pm 0,08^{\mathrm{efg}}$ \\
& Etanol & $4,5 \pm 0,5^{\mathrm{d}}$ \\
& Metanol & $11,1 \pm 0,4^{\mathrm{c}}$ \\
& Água/Etanol $(30 / 70)$ & $16,7 \pm 0,2^{\mathrm{b}}$ \\
Água/Etanol (50/50) & $20,5 \pm 0,8^{\mathrm{a}}$ \\
\hline
\end{tabular}

(1) média \pm desvio padrão $(\mathrm{n}=2)$; Letras iguais na mesma coluna indicam que não há diferença significativa $(\mathrm{p}$ $>0,05)$

A Tabela 2 apresenta os resultados médios de rendimento global de extração $\left(\mathrm{X}_{0}\right)$ dos experimentos realizados por ESC com tansagem ( $P$. major) e as condições de temperatura e pressão utilizadas nos ensaios. Os dados correspondentes de massa específica $(\rho)$ do $\mathrm{CO}_{2}$ também estão apresentados na Tabela 2 .

A temperatura e a pressão são fatores que afetam tanto o rendimento de extração como a classe de compostos extraídos. O poder de solvatação do solvente supercrítico é afetado pela pressão e temperatura de operação devido a sua ação na densidade do solvente. Consequentemente, o poder de solvatação pode ser modificado por meio da manipulação da pressão e temperatura de operação. Como regra geral, a pressão constante, um aumento de temperatura diminui a densidade do solvente $\mathrm{e}$ seu poder de solvatação, enquanto que a temperatura constante, um aumento da pressão aumenta a densidade do fluido supercrítico (Brunner, 1994). Dessa forma, o aumento no rendimento de extração da tansagem ( $P$. major) a temperatura constante pode ser atribuído ao aumento da densidade do fluido supercrítico decorrente do aumento da pressão.

A solubilidade dos solutos no solvente supercrítico é dependente, além dos fatores temperatura e pressão, da massa molar, polaridade e pressão de vapor. Dessa maneira, o efeito da temperatura na solubilidade é complexo devido a combinação de dois fatores: o incremento na temperatura aumenta a pressão de vapor do soluto, aumentando sua solubilidade no solvente supercrítico, no entanto, ao 
mesmo tempo ocasiona a diminuição da densidade do solvente. Os efeitos antagônicos da densidade do solvente e da pressão de vapor do soluto podem ser observados na Figura 1, a qual apresenta as isotermas de rendimento do extrato de tansagem ( $P$. major) em $\mathrm{CO}_{2}$ supercrítico como função da pressão de operação, de acordo com os resultados da Tabela 2, para as temperaturas de 40,50 e $60{ }^{\circ} \mathrm{C}$.

Tabela 2 - Rendimento global $\left(\mathrm{X}_{0}\right)$ obtidos para as extrações com dióxido de carbono supercrítico de tansagem (P. major)

\begin{tabular}{lccc}
\hline Temperatura $\left({ }^{\mathrm{o}} \mathrm{C}\right)$ & Pressão $(\mathrm{MPa})$ & $\rho \mathrm{CO}_{2}\left(\mathrm{~g} / \mathrm{cm}^{3}\right)$ & Rendimento $(\%)^{(1)}$ \\
\hline 40 & 10 & 0,629 & $0,62^{\mathrm{c}} \pm 0,01$ \\
40 & 20 & 0,840 & $0,8^{\mathrm{b}} \pm 0,1$ \\
40 & 30 & 0,911 & $0,87^{\mathrm{ab}} \pm 0,04$ \\
50 & 10 & 0,385 & $0,59^{\mathrm{c}} \pm 0,02$ \\
50 & 20 & 0,785 & $0,81^{\mathrm{b}} \pm 0,02$ \\
50 & 30 & 0,871 & $0,97^{\mathrm{a}} \pm 0,03$ \\
60 & 10 & 0,295 & $0,57^{\mathrm{c}} \pm 0,02$ \\
60 & 20 & 0,724 & $0,80^{\mathrm{b}} \pm 0,02$ \\
60 & 30 & 0,830 & $0,99^{\mathrm{a}} \pm 0,01$ \\
\hline
\end{tabular}
$(\mathrm{p}>0,05)$

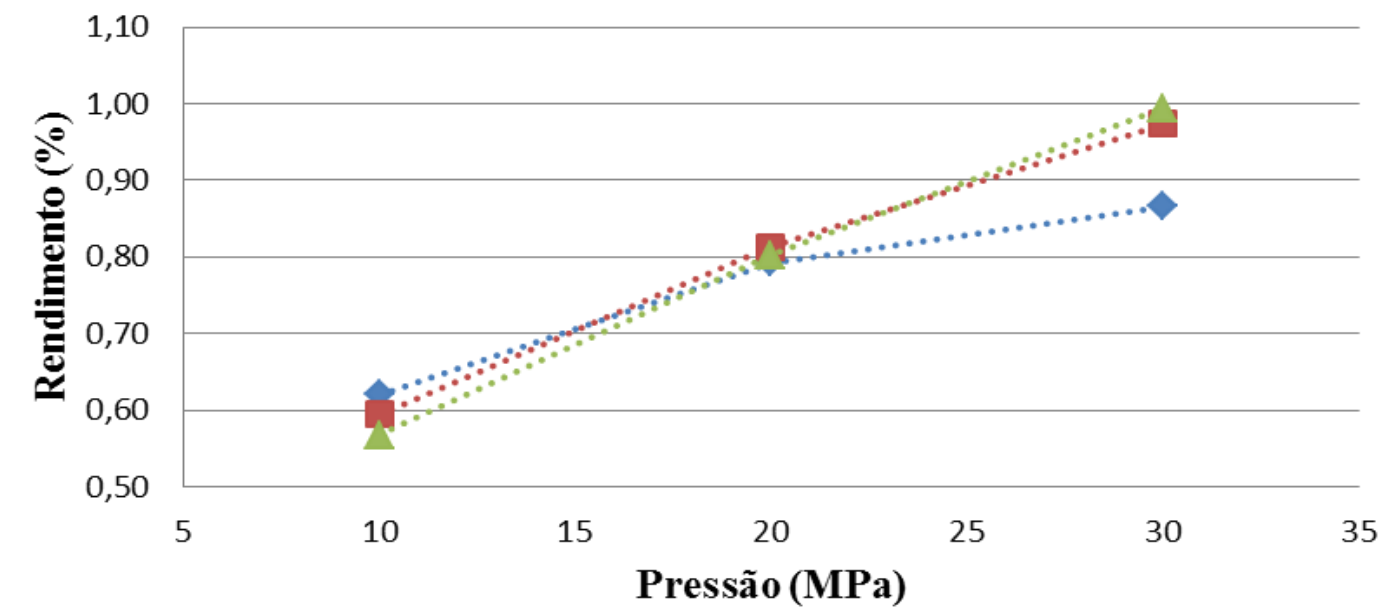

$\cdots \bullet$ Temperatura $40{ }^{\circ} \mathrm{C} \quad \cdots \cdot \cdot$ Temperatura $50{ }^{\circ} \mathrm{C} \quad \cdots * \cdot$ Temperatura $60{ }^{\circ} \mathrm{C}$

Figura 1 - Comportamento das isotermas de rendimento com a pressão de extração para a ESC de tansagem (Plantago major).

Em baixas pressões a solubilidade diminui com o aumento da temperatura uma vez que a 
densidade do solvente decresce rapidamente com o aumento da temperatura; a altas pressões as mudanças de densidade com a temperatura são muito mais discretas, de modo que o aumento na pressão de vapor causado pelo aumento de temperatura torna-se mais importante do que a ligeira diminuição de densidade (Brunner, 1994). Esses dois efeitos ocasionam uma inversão da curva de solubilidade, fenômeno conhecido como retrogradação, que é resultado da predominância de um dos dois fatores (Michielin et al., 2005).

$\mathrm{O} \mathrm{CO}_{2}$ tem uma limitada habilidade de extrair compostos de maior polaridade, como é a maioria das substâncias com propriedades antioxidantes, mesmo quando operado em condições que propiciem altas densidades. Dessa forma, apesar de todas as vantagens da utilização do dióxido de carbono em processos de extração supercrítica, o fato de o $\mathrm{CO}_{2}$ ser um composto apolar torna o processo pouco eficiente. Esse fato pode ser observado, comparando-se os rendimentos obtidos por ESC com $\mathrm{CO}_{2}$ supercrítico puro aos resultados obtidos nas extrações convencionais (Tabelas 1 e 2), onde as extrações com solventes orgânicos apresentaram rendimentos superiores. Esses resultados podem ser atribuídos, entre outros fatores, a polaridade dos solventes empregados nas extrações.

Com o objetivo de aumentar o rendimento de extração de compostos polares, fez-se o uso de um modificador. A adição de modificadores ao $\mathrm{CO}_{2}$ supercrítico pode aumentar a eficiência de extração, uma vez que aumenta a solubilidade dos compostos. Para a escolha do solvente, levaram-se em consideração os resultados de rendimentos obtidos nos processos de extração a baixas pressões e, além disso, estabeleceu-se que o mesmo deveria ser um modificador de grau alimentício.

Os resultados obtidos para ESC com etanol como cossolvente estão representados na Figura 2. A adição de cossolvente ao processo de extração supercrítica com $\mathrm{CO}_{2}$ elevou os valores de rendimento de extrato. A adição de $10 \%$ de cossolvente proporcionou um incremento de 2,3 vezes o rendimento obtido.

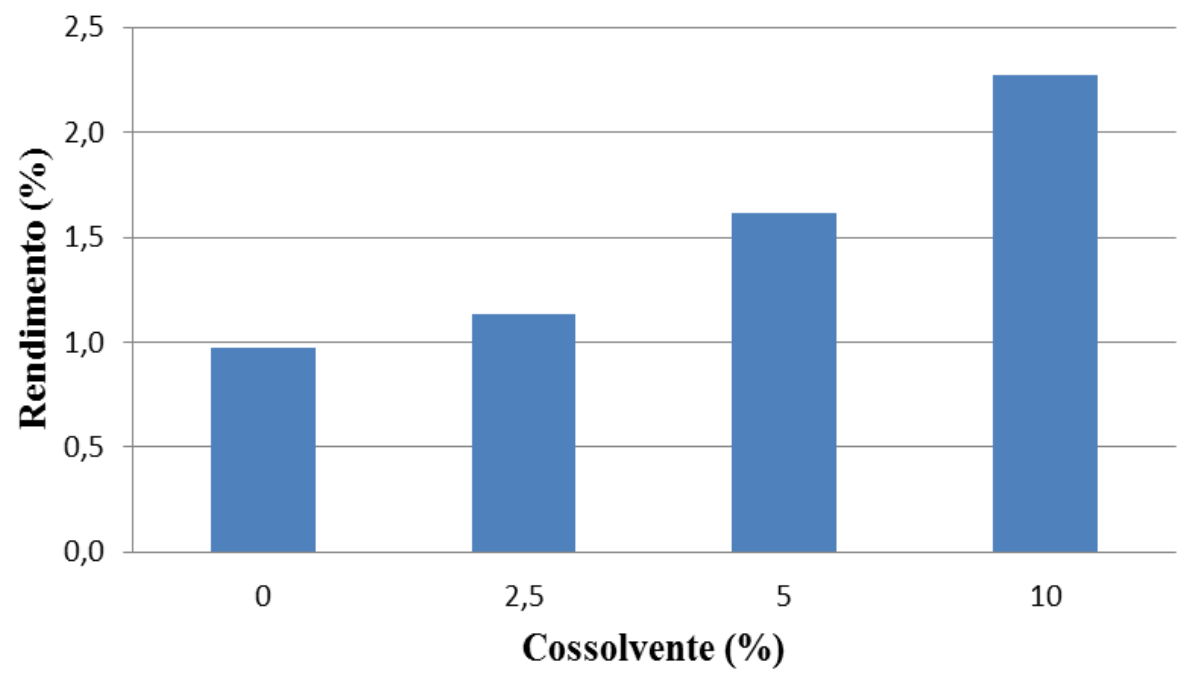

Figura 2 - Rendimento global $\left(\mathrm{X}_{0}\right)$ para os extratos de tansagem (P. major) utilizando etanol como cossolvente. 


\section{9 a 22 de outubro de 2014 \\ Florianópolis/SC}

\section{CONCLUSÕES}

O maior rendimento de extração $(20,5 \pm 0,8 \%)$, dentre as diferentes tecnologias aplicadas a extração de tansagem, foi obtido por ultrassom empregando a mistura de etanol/água (50/50) como solvente. A extração supercrítica apresentou rendimentos de extração inferiores aos rendimentos de extração com Soxhlet e ultrassom, sendo o maior obtido a $30 \mathrm{MPa}$ e $60{ }^{\circ} \mathrm{C}(0,99 \pm 0,01 \%)$. O emprego de etanol como cossolvente ao processo de extração supercrítica aumentou o rendimento em 2,3 vezes.

Avaliando as tecnologias aplicadas quanto a rendimento de extração, verificou-se que a técnica por ultrassom apresentou os melhores resultados. No entanto, a qualidade biológica desses extratos deve ser analisada para definir qual o melhor método a ser utilizado nas extrações de tansagem $(P$. major).

\section{AGRADECIMENTOS}

Os autores agradecem a CAPES pelo auxilio financeiro.

\section{REFERÊNCIAS}

ANDRADE, K.S.; GONÇALVEZ, R.T.;MARASCHIN, M.; RIBEIRO-DO-VALLE, R.M.; MARTÍNEZ, J,; FERREIRA, S.R. Supercritical fluid extraction from spent coffee grounds and coffee husks: Antioxidant activity and effect of operational variables on extract composition. Talanta, v. 88, n. 0, p. 544-552, 2012.

AOAC. Association of Official Analytical Chemists - Official Methods of Analysis of the AOAC International. 18. ed. Maryland: AOAC, 2005.

BLANCO, B.; SABORÍO, A.; GARRO, G. Descripción anatómica, propiedades medicinales y uso potencial de Plantago major llantén mayor. Tecnología en Marcha, v. 21, n. 2, p. 17-24, 2008.

BRUNNER, G. Gas Extraction: An introduction to fundamentals of supercritical fluids and the applications to separation process. Darmastadt, Alemanha: Steikopff, Springer, 1994. 387p.

CECHINEL FILHO, V.; YUNES, R. A. Estratégias para a obtenção de compostos farmacologicamente ativos a partir de plantas medicinais: conceitos sobre modificação estrutural para otimização da atividade. Química Nova, v. 21, p. 99-105, 1998.

CHÁECIA. Tanchagem. Disponível em:<http://www.chaecia.com.br/loja/produto-111058-1339tanchagem_plantago_major_1_100_grg>. Acesso em: 1 nov. 2013.

GÁlVEZ, M.; MARTÍN-CORDERO, C.; HOUGHTON, P. J.; AYUSO, M. J. Antioxidant Activity of Methanol Extracts Obtained from Plantago Species. Journal of Agricultural and Food Chemistry, v. 53, n. 6, p. 1927-1933, 2005 
GIL-CHÁVEZ, J. G.; VILLA, J.A.; AYALA-ZAVALA, J.F.; HEREDIA, J.B.; SEPULVEDA, D.; YAHIA, E.M.; GONZÁLEZ-AGUILAR, G.A. Technologies for Extraction and Production of Bioactive Compounds to be Used as Nutraceuticals and Food Ingredients: An Overview. Comprehensive Reviews in Food Science and Food Safety, v. 12, n. 1, p. 5-23, 2013.

MICHIELIN, E. M. Z.; BRESCIANI, L. F. V.; DANIELSKI, L.; FERREIRA, S. R. S.

Composition profile of horsetail (Equisetum giganteum L.) oleoresin: comparing SFE and organic solvents extraction. Journal of Supercritical Fluids, v. 33, p. 131-8, 2005.

PEREIRA, C.; MEIRELES, M. Supercritical Fluid Extraction of Bioactive Compounds:

Fundamentals, Applications and Economic Perspectives. Food and Bioprocess Technology, v. 3, n. 3, p. 340-372, 2010.

SAMUELSEN, A. B. The traditional uses, chemical constituents and biological activities of Plantago major L. A review. Journal of Ethnopharmacology,v. 71, n. 1-2, p. 1-21, 2000. 